\title{
Motivación del logro y resolución de problemas con funciones lineales en una universidad de Surco
}

\author{
Gean Pierre Rostaing Ccapacca ${ }^{1 a}$, Luis Alexis Bernuy Torres², César Andrés Borja Villanueva ${ }^{3}$, \\ Edwin Alberto Martínez López ${ }^{4}$, María Fernanda Ayllón Blanco ${ }^{5}$ y Isabel Angustias Gómez \\ Pérez ${ }^{6}$
}

Universidad Peruana de Ciencias Aplicadas, Lima, Perú ${ }^{1}$ Universidad Privada Juan Pablo II, Lima, Perú ${ }^{23}$ Universidad Cesar Vallejo, Lima, Perú ${ }^{4}$

Centro de Magisterio La Inmaculada, Granada, España ${ }^{56}$

Orcid ID: https://orcid.org/0000-0001-8383-9968

Orcid ID: https://orcid.org/0000-0001-9011-7699²

Orcid ID: https://orcid.org/0000-0002-4274-49783

Orcid ID: https://orcid.org/0000-0002-1769-11814

Orcid ID: https://orcid.org/0000-0001-6690-2742

Orcid ID: https://orcid.org/0000-0001-9941-3099

\section{Recibido: 04 de febrero de 2018}

Aceptado: 12 de octubre 2018

\section{Resumen}

La presente investigación tuvo como objetivo determinar la relación entre la motivación del logro y la resolución de problemas con funciones lineales de los estudiantes de Fundamentos para el Cálculo del turno tarde de la Facultad de Negocios en la Universidad Peruana de Ciencias Aplicadas, 2017. Este estudio se desarrolló dentro de un enfoque cuantitativo, con un método hipotético deductivo y diseño no experimental. La muestra estuvo conformada por 76 estudiantes de Fundamentos para el Cálculo de la Facultad de Negocios de la universidad mencionada. Los resultados permitieron determinar que no existe relación directa y significativa entre la motivación del logro y la resolución de problemas con funciones lineales de los estudiantes encuestados. Esto demostró que la competencia de resolver problemas no depende de la motivación, sino más bien se requiere de conocimiento y habilidades que se manejen.

Palabras clave: motivación y resolución de problemas. 


\title{
Motivation of achievement and problem solving with linear functions at a university in Surco
}

\begin{abstract}
The objective of this research study was to determine the relationship between the motivation of achievement and the resolution of problems with linear functions of the students of Foundations of Calculus for the afternoon course of the Faculty of Business at the Peruvian University of Applied Sciences, 2017. This study was developed within a quantitative approach, with a hypothetical deductive method and non-experimental design. The sample consisted of 76 students of Foundations of Calculus from the Faculty of Business of the aforementioned university. The results show that there is no direct and significant relationship between the motivation of achievement and the resolution of problems with linear functions of the students surveyed. This further showed that the competency to solve problems does not depend on motivation, but rather requires knowledge and skills that are put into practice.
\end{abstract}

Keywords: motivation and problem solving.

\section{Introducción}

En muchas ocasiones se dice que hacer matemática es solucionar problemas, pues dicha afirmación es correcta hasta un cierto punto. En el marco internacional se le otorga gran importancia a la resolución de problemas en la enseñanza de las matemáticas. El Programa Internacional para la Evaluación de Estudiantes (PISA) realizó una prueba en el 2012, donde los resultados demostraron que el $20 \%$ de los estudiantes solo es capaz de solucionar problemas muy simples, como las situaciones familiares a ellos. Además, se conoció que el 50\% de los estudiantes, en muchos países de la región, no sabía escribir o leer apropiadamente y no tenían los conocimientos más básicos en matemáticas. Por otro lado, en Japón y Corea del Sur, más del 90\% de estudiantes puede terminar tareas de nivel 2. Los países mencionados se encuentran cerca de obtener el objetivo fundamental de que cada alumno tenga las herramientas básicas suficientes para enfrentar los diversos retos desconocidos de la vida diaria.

Se dice que una persona que sabe matemática es capaz de resolver problemas, no solo matemáticos, sino de la vida diaria. Polya (1965) afirma que la resolución de problemas es un barómetro del conocimiento matemático. No obstante, para saber resolver problemas es necesario aprender. Ayllón, Gómez y Ballesta (2016) recogen de Brown (1978) que al igual que se ha de aprender determinados conceptos, o a memorizar o a realizar un algoritmo se ha de aprender a resolver problemas, los cuales ayudarán a interiorizar los aprendizajes enumerados. 
La prueba PISA (2012) demostró que el 20\% de los estudiantes resuelve problemas muy sencillos o que sean familiares. Además, el $50 \%$ de los estudiantes no entiende lo que lee; por lo que no son capaces de resolver problemas a diferencia de los estudiantes de Corea del Sur y Japón, donde muchos de ellos tienen un alto nivel matemático y son capaces de afrontar nuevos retos.

A nivel nacional, muchos alumnos peruanos se encuentran en un nivel 1 o por debajo del mismo en rendimiento de las escalas matemáticas (75\%), ciencias (69\%) y lectura (60\%), lo cual muestra una deficiencia académica. El informe de PISA (2015) describe y da ejemplos de lo que esto significa en términos de las habilidades de los alumnos. Entonces, podría decirse que los estudiantes peruanos solo pueden solucionar problemas simples, y en algunas ocasiones ni eso.

Sumado a ello, PISA demostró la desigualdad del sistema peruano en educación. Por ejemplo, hay mucha diferencia entre el rendimiento de los estudiantes rurales con los urbanos. A favor de estos últimos mencionados, los docentes son muy tradicionales y se has dedicado a solo el "dictado" de clases. En la actualidad, el Perú se ubica en el puesto 64 del ranking mundial en rendimiento de ciencias, lectura y matemática, según los resultados PISA del 2015, notándose una mejora.

El estudiante peruano, según estudios PISA (2015), en general tiene un bajo rendimiento matemático. Esto se debe a muchos factores; tales como la desigualdad, el poco enfoque que el docente le otorga a la resolución de situaciones problemáticas y la escasez de recursos pedagógicos. Noda (2000, citado en Ayllón 2012) asegura que la resolución de problemas es la pieza clave para construir el conocimiento matemático siendo una actividad cognitiva básica para el desarrollo integral de la persona.

La resolución de problemas, dentro de la matemática, es fundamental para la formación de los estudiantes en el ámbito profesional y personal. De esta manera, podrán afrontar los diversos problemas que se dan en la vida diaria. En la Universidad Peruana de Ciencias Aplicadas se nota la poca motivación en el curso de matemática y los pocos recursos que tienen los estudiantes para poder resolver problemas. Sin embargo, no habría prueba de ello si no se toma en cuenta estudios previos y los resultados de un proceso estadístico.

Erazo (2015), desarrolló una investigación con el fin de crear una guía didáctica virtual de estrategias motivacionales en la enseñanza de la matemática para los docentes de educación general básica. Los resultados demostraron que los estudiantes casi nunca 
se esfuerzan por realizar sus metas, remarcando la escasez de motivación intrínseca por parte de ellos y extrínseca por parte del docente.

Navea (2015) analizó el nivel los alumnos universitarios de ciencias de la salud, específicamente los de fisioterapia y enfermería, como se encuentran motivados y autorregulados, cuáles son las estrategias motivacionales y de aprendizaje más importantes que aplican en su periodo universitario. Los resultados permitieron observar que muchos de ellos tienen una baja motivación por la carrera, a pesar de que muchos muestran interés desde pequeños. Este poco interés se debe a que no cuentan con buenas estrategias motivacionales del aprendizaje y por ello no se autorregulan.

Jiménez (2015) busco encontrar la existencia de la relación entre la motivación y aprendizaje del curso de contabilidad en estudiantes del primer ciclo de administración 2015. Se identificó que el principal problema que afrontan las universidades mundiales es el poco interés por aprender, que no es más que la ausencia de motivación que presentan los educandos para superarse académicamente. Se concluyó que todo estudiante requiere de ser motivado para lograr, y en algunos casos, mejorar su aprendizaje.

Sánchez (2013) tuvo como objetivo encontrar la relación entre la motivación de logro y el rendimiento académico del primer ciclo de la especialidad de contabilidad en el Instituto de Formación Bancaria (IFB) 2013 del distrito de Los Olivos. Concluyó que la motivación de logro no se relaciona de forma significativa con el rendimiento académico, en estudiantes del primer ciclo de la especialidad de contabilidad en el IFB.

En la misma línea Gasco y Villarroel (2014) en su estudio "La motivación en la Resolución de problemas aritmético-algebraicos: Un estudio con alumnado de secundaria" aseguran que aunque la motivación influye de manera importante en el aprendizaje matemático, no queda evidenciado si la motivación refleja la implicación del alumno en matemáticas, ya que al realizar la revisión bibliográfica para su investigación, no encuentran investigaciones significativas que relacionen la resolución de problemas con la motivación.

Posteriormente, Ruiz y Quintana (2016) llevan a cabo una investigación sobre la relación entre la Atribución de Motivación de logro y el rendimiento académico en matemática. Este estudio se realizó con 993 estudiantes de secundaria. En él sí aparecen evidencias de que el rendimiento académico en la asignatura de matemática está vinculado con el interés, el esfuerzo, así como con la implicación y formación del docente. 


\section{Motivación de logro}

Atkinson y Feather (1974) afirmaron que la motivación de logro:

Es el comportamiento en contextos como los académicos y profesionales en que la conducta está orientada hacia la consecución de logros como el aprendizaje o los éxitos propios del ámbito laboral está determinado por el deseo de éxito, el miedo al fracaso y, eventualmente, por motivaciones externas a los logros intrínsecos a la actividad a realizar (p. 2).

Esta afirmación define a la motivación del logro como motor principal del comportamiento estudiantil, en el ámbito educativo, pues ésta guía a la obtención de la meta, que es el aprendizaje. Dentro de la motivación del logro hay componentes, como el miedo a quedar mal frente a otros, el deseo de hacer las cosas bien y en algunos casos, los estímulos externos del individuo. Por ejemplo, cuando un estudiante desea salir a la pizarra a realizar un problema para demostrar lo que sabe o cuando un estudiante muestra sus buenas calificaciones, para obtener una satisfacción personal y reconocimiento de otros.

Según Murray (1938) "la motivación del logro se refiere al esfuerzo por desempeñar las tareas difíciles tan bien como sea posible, así como otras necesidades fisiológicas y psicológicas que también contribuyen al desarrollo de la personalidad". Esta definición, desde el punto de vista cognitivo, muestra un deseo de superación que nace dentro del ser, pues afirma que la motivación por obtener un objetivo es el trabajo que realiza el individuo de forma eficiente.

Por su parte; Mc Clelland, Atkinson, Clark y Lowell (1953) afirmaron que la motivación del logro "es aquella motivación aprendida a través de la interacción social, que mueve a la persona a conducir sus esfuerzos y acciones, de manera persistente en busca de un desempeño exitoso que alcance o sobrepase estándares de excelencia”.

Dichos autores argumentaron que la motivación del logro se aprende a través de las relaciones sociales, podría decirse que es un aprendizaje sociocultural. Esto, ya que genera en el individuo un sobreesfuerzo de forma permanente al realizar una tarea, teniendo como meta obtener un óptimo desempeño y alcanzar un nivel superior.

Además, Atkinson y Birch (citados en Soriano, 2001) señalaron que la motivación del logro "es una motivación que empuja y dirige la consecución exitosa de forma competitiva, de una meta u objetivo reconocido socialmente" (p. 10). Al igual que muchos autores, ambos definieron a la motivación del logro como motivación en sí, fuente de inspiración que encamina a obtener lo que se anhela. Por ejemplo, la 
satisfacción personal de quedar bien frente a otras personas, el reconocimiento y la aceptación social o alguna recompensa externa que satisfaga alguna necesidad del ser.

El motivo de logro o motivación del logro, el cual fue estudiado inicialmente por Murray (1938) y desarrollado posteriormente por McClelland (1989) "es la tendencia a buscar el éxito en tareas que implican la evaluación del desempeño". Según Chóliz (2004), este deseo o afán de obtener el éxito, la disposición estable que tiene una persona en su conducta, y la competitividad natural de cada persona, se forma durante la infancia (p. 46). Desde su postura, Bengochea (1996) afirmó que la motivación al logro "es el deseo de sobresalir, de triunfar o alcanzar un nivel de excelencia, está relacionada con la persistencia, cantidad y calidad del trabajo" (p. 59).

Murray y Bengochea, coincidieron y argumentaron que la motivación del logro es anhelar y dirigirse hacia el éxito. Esta búsqueda genera una competencia y una evaluación del rendimiento, para así poder esforzarse continuamente y mejorar considerablemente hasta alcanzar un nivel óptimo.

Sin embargo, parecen ser ciertas las palabras de Pozo y Monereo (2001), quienes afirmaron que en los colegios actualmente se dictan contenidos del siglo XIX, con docentes del siglo XX, a estudiantes del siglo XXI. Bajo dicha afirmación, se muestra un problema motivacional relacionado al contenido académico y al método de enseñanza.

Para ahondar en el primer punto, la metodología de la enseñanza está dado por el docente que lamentablemente, en muchos casos, no se genera interés en el alumno. El segundo punto es el aprendizaje basado en el estudiante, donde se debe considerar las variaciones educativas que se han producido en la actualidad. Estos cambios han transformado la perspectiva hacia el aprendizaje y el rol del estudiante dentro de este proceso. El centro de atención antiguamente era el docente, pero en la actualidad es el estudiante, pues este genera su propio aprendizaje. Este es quien construye sus conocimientos y no reproduce de forma mecánica lo que se enseña como antes. Bajo esta óptica, la motivación del estudiante deja de situarse solo de forma extrínseca, más bien se sitúa dentro del estudiante. Por tanto, este sería un segundo problema motivacional.

El aprendizaje basado en el estudiante es el segundo factor motivacional, pues se han visto cambios en la educación. Desde el punto de vista cognitivo, el estudiante construye su propio aprendizaje. Por ello, la motivación también ha cambiado, pues ya no parte de una motivación externa, sino que depende del propio estudiante. 
La cultura social es el último factor, pues la falta de motivación también está relacionada a otros puntos, más allá del docente y alumno. Es un factor situado en otro nivel, pues es más complejo accionar sobre esta. Son determinantes de clase cultural; como la forma de vivir, la influencia de las tecnologías, la formación de valores, las relaciones sociales, etc. Estos componentes socioculturales afectan la motivación de los alumnos, lo cual genera un nuevo problema motivacional diferente a las anteriores (Núñez, 2009).

El tercer factor, además de la enseñanza y el aprendizaje, es la cultura. Este punto está situado en otra escala, diferente a las dos anteriores, pues es más difícil actuar sobre esta. Se refiere a los cambios culturales que afectan a la sociedad durante los últimos años y está relacionado a los valores, las tecnologías, las relaciones intrapersonales e interpersonales, etc. Estos aspectos socioculturales influyen en la escaza motivación estudiantil, la cual genera otro problema motivacional distinto al resto.

\section{Resolución de problemas}

La resolución de problemas matemáticos es importante para la vida del hombre, no solo en el ámbito matemático, sino en general. Por ello, Polya (citado en Boscan y Klever, 2012) señaló que resolver un problema "es encontrar un camino allí donde no se conocía previamente camino alguno, encontrar la forma de sortear un obstáculo, conseguir el fin deseado, que no es conseguible de forma inmediata, utilizando los medios adecuados" (p. 11).

En la vida diaria, existen diversos problemas, además de matemáticos, los cuales pueden no contar con soluciones tan sencillas, pero el análisis de esta dificultad prepara a las personas a enfrentar e idear la manera de solucionarlo, generando así personas autosuficientes.

Según Schoenfeld (1998, citado en Iriarte y Sierra, 2011), resolver un problema:

Es analizar una situación que precisa una solución pero que, generalmente, no tiene un camino de solución rápido y directo, sino que debe ir realizando una toma de decisiones (y, por ende, modificando y comprobando) a lo largo de la propia resolución (p. 49).

Según lo referido, al momento de resolver un problema, se están realizando procesos de toma de decisiones, pues cada paso que se realiza tiene que tener coherencia y verificar lo anterior, debe seguir una secuencia lógica. Llevando esto a la realidad, muchas personas toman diariamente malas decisiones. Esto se debe a no analizar las 
consecuencias antes de actuar, y en algunos casos toman decisiones incoherentes a lo que se dice.

Desde la postura de Parra (1990), la resolución de problemas "es la coordinación de experiencias previas, conocimiento e intuición, en un esfuerzo para encontrar una solución que no se conoce" (p. 15). A diferencia de dicho autor; Marson, Burton y Stace (1989) afirmaron que la resolución de problemas "es un proceso dialectico, donde las tareas pueden avanzar o retroceder" (p. 63).

En tal sentido, Parra reafirmó las definiciones de los autores nombrados anteriormente, pero incluye la intuición como componente en la resolución de un problema. Pues, más que la intuición se debe incluir la suerte al momento de solucionar una situación.

Con respecto a la resolución de problemas matemáticos, Guzmán (2017), manifestó que la resolución de problemas en la enseñanza de las matemáticas:

Es la intención de transmitir, de una manera sistemática, los procesos de pensamiento eficaces en la resolución de verdaderos problemas. Tal experiencia debe permitir al estudiante activar su capacidad mental, ejercitar su creatividad y reflexionar sobre su propio aprendizaje (metacognición) al tiempo que se prepara para otros problemas, con lo que adquiere confianza en sí mismo (p. 11).

En conclusión, al solucionar un problema en matemática, se está construyendo el razonamiento en el estudiante, quien explotará su capacidad cognitiva, para mejorar su creatividad y volverse un estudiante autónomo. La resolución del problema no solo genera capacidades cognitivas, también genera automotivación y confianza en el estudiante, elemento importante para enfrentarse posteriormente a otras actividades similares.

Por otro lado, Iriarte y Sierra (2011) definieron que la resolución de problemas "es un proceso mental, donde influyen habilidades, competencias, conocimientos tanto declarativos (por cuanto puede comunicarse o declararse a través del lenguaje verbal), procedimentales como actitudinales" (p. 58).

Un estudiante, al momento de resolver un problema, está demostrando sus habilidades cognitivas y su carácter; pues solucionar un problema requiere tener una personalidad adecuada. Por ello, algunos estudiantes poseen conocimientos respecto al problema, pero a veces no es capaz de resolverlo por la ausencia de motivación al enfrentarse a una tarea, por lo que tienden a abandonar el problema por su deficiencia actitudinal.

Actualmente se observa, en el área de matemática, nuevos planteamientos curriculares, los cuales buscan mejorar la enseñanza matemática. Esto, ya que es 
considerada muy difícil y de bajos resultados académicos por todo el mundo. Una nueva propuesta es centrar el proceso de enseñanza-aprendizaje de las matemáticas en la solución de problemas, propuesta que se está implementando en el Perú.

Antiguamente, enseñar matemática era centrarse en resolver miles de ejercicios y básicamente aprender por repetición, donde en algunos casos se trabaja mecánicamente sin analizar la solución. Esto ha dado un giro didáctico, donde se plantean nuevas formas de generar el aprendizaje en los estudiantes, relacionando sus experiencias y la capacidad de tomar decisiones ante diversos casos, por medio del aprendizaje basado en problemas y en su resolución de estos.

La enseñanza de matemática está avanzando, pero no por la acumulación de contenidos, sino por la implementación de resolución de problemas, para una mejor comunicación y comprensión matemática, tanto en forma individual como colectiva. Para saber matemática se tiene que aplicar matemática, de tal manera que se llegue a la abstracción, organización, análisis, representación, clasificación, generalización, diferenciación, predicción, etc.

El mecanicismo clásico para aprender matemática, en definitiva, se quedó años atrás. Ahora, la creatividad del estudiaste es pieza fundamental para la resolución de problemas, los cuales han sido clasificados por Blanco (1991), teniendo en cuenta que, todos los diversos problemas se pueden plantear en diversos contextos, niveles y mezcladas entre sí (p. 31). Partiendo de esta afirmación, la clasificación de problemas se puede expresar de ocho maneras.

Los problemas de reconocimiento son aquellos ejercicios que simplemente buscan identificar y recordar alguna propiedad, teorema o definición ya estudiada, donde se establecen comparaciones, relaciones, argumentaciones y justificaciones en los enunciados. Por ejemplo: ¿Es cierto que $2+5<8-3$ ?, si " $\mathrm{x}$ ” es un número positivo e “y” es número negativo ¿El producto de estos números definidos es negativo?, o identificar el error en una operación matemática con la finalidad de obtener conclusiones.

Los problemas de tipo algorítmico son aquellos ejercicios que se pueden solucionar por medio de una secuencia algorítmica, que ya se conoce y solo se aplica, donde no hay una exigencia matemática en desarrollar la habilidad creativa del estudiante. Por ello, se busca plantear estos problemas en diversas situaciones como gráficas, tablas, etc. Algunos ejemplos de estos son la resolución de una ecuación cuadrática o el despeje de una incógnita en una ecuación. 
Un tipo de problema, donde los estudiantes muestran mayores deficiencias, es el de traducción, donde el estudiante busca convertir un lenguaje usual a un lenguaje matemático. También, busca representar una expresión, para luego resolverla matemáticamente por medio del cálculo. Este tipo de problema requiere una buena organización e interpretación para la compresión del problema, así buscando plantear una expresión, para luego solucionarla. Por ejemplo: en una fiesta hay 25 personas, sabiendo que la relación entre hombres y mujeres es como 3 es a 2. ¿Cuántos hombres hay en la reunión?

Los problemas relacionados a situaciones reales son muy frecuentes en las universidades, pues requieren una buena organización de datos, una representación e interpretación de estos, finalizando con el cálculo y el análisis de los resultados obtenidos ante una situación problemática. Estos problemas, muchas veces, requieren un conocimiento general aparte del matemático, por ello su complejidad. Por ejemplo: si la oferta y la demanda de una empresa están definidas de la siguiente manera “ $p=2 q+30$ " $\mathrm{y}$ “ $p=-4 q+70 ”$ ¿A partir de qué precio se genera un exceso de oferta?

Los problemas que demuestran ciertos comportamientos o regularidades son los problemas de investigación matemática, los cuales se caracterizan por actividades lógicas, demostraciones, probar algún teorema encontrando su validez. Para realizar este tipo de resoluciones, se requiere un alto nivel cognitivo matemático. Por ejemplo: demostrar que, entre tres términos de una progresión geométrica, el término central es la media geométrica de los otros dos.

Los problemas de tipo puzles son aquellos que buscan potencializar las habilidades creativas en los estudiantes. La forma de resolver este tipo de problema depende de las perspectivas que se planteen. Los puzles no requieren necesariamente un proceso matemático, podría decirse, que se pueden resolver mediante una idea inesperada. Por ejemplo: partir en triángulos acutángulos un triángulo rectángulo.

Por último, se encuentran los problemas de tipo historias matemáticas, los cuales, por medio de la literatura, algunos autores pueden expresar sus conocimientos matemáticos. Por ejemplo, en los viajes de Gulliver, donde se plantean la proporcionalidad y semejanza. La finalidad de estos problemas es generar la curiosidad e interés al campo matemático. 


\section{Método}

La presente investigación se desarrolló dentro del enfoque cuantitativo, el cual tiene como finalidad explicar un fenómeno, para predecirlo y controlarlo en un futuro (Guba y Lincoln, 1994). Por su parte, Ramos (2015) afirmó que la investigación cuantitativa:

Es la utilización de los datos recolectados para poner a prueba o comprobar las hipótesis mediante el uso de estrategias estadísticas basadas en la medición numérica, lo cual permitiría al investigador proponer patrones de comportamiento y probar los diversos fundamentos teóricos que explicarían dichos patrones (p. 12).

Se puede entender que la investigación cuantitativa trata de explicar las diversas situaciones en el mundo real de una forma exacta y precisa, la cual primero recolecta información del fenómeno que se desea estudiar, lo procesa por medio de aplicaciones estadísticas, luego organiza la información en tablas y gráficos y genera relaciones, dándole sentido a los fundamentos teóricos y formando patrones generales, para luego predecir ante una situación en común.

El diseño no experimental es aquel que se realiza sin la manipulación y control de las variables estudiadas. En este diseño de investigación no hay estímulos ni condiciones a las cuales se exponga la muestra. Según Gonzales (2012) la investigación no experimental:

Es la investigación sistemática y empírica en las que las variables independientes no se manipulan porque ya han sucedido. Las influencias sobre las relaciones entre variables se realizan sin intervención o influencia directas y dichas relaciones se observan tal y como se han dado en su contexto natural (p. 3).

En tal afirmación, se puede concluir que el diseño no experimental se realiza únicamente cuando las variables están como tal, sin la participación de ningún ser que pueda controlar deliberadamente alguna variable estudiada. Este diseño analiza las variables en un momento dado, encontrando la relación que guardan estas o la evolución que tiene una con respecto a la otra. En la cual podemos definir dos cortes, longitudinal y transversal.

\section{Resultados}

De acuerdo a la hipótesis general; existe relación entre la motivación de logro y la resolución de problemas con funciones lineales en los estudiantes de Fundamentos para el Cálculo del turno tarde de la Facultad de Negocios en la UPC, 2017; el valor de $\alpha$ es 
0.05, llamado nivel de significación teórica, lo cual corresponde al nivel de confianza con que se trabaja que es un $95 \%$.

Tabla 1

Coeficiente de correlación entre la motivación de logro y la resolución de problemas con funciones lineales de los estudiantes de fundamentos para el cálculo del turno tarde de la facultad de Negocios en la UPC 2017

\begin{tabular}{lcc}
\hline & & Resolución de problemas \\
\hline & Correlación de Spearman & -.094 \\
Motivación de logro & Sig. $(p)$ & .420 \\
& $\mathrm{~N}$ & 76 \\
\hline Fuente: Base de datos & &
\end{tabular}

No existe relación entre ambas variables.

Debido a que el valor obtenido por el coeficiente de correlación de Spearman es $\mathrm{p}=0.420$ y supera al valor de significación teórica $\alpha=0.05$, no se rechaza la hipótesis nula. Esto demuestra que no existe relación directa y significativa entre la motivación de logro y la resolución de problemas con funciones lineales de los estudiantes de Fundamentos para el Cálculo del turno tarde de la Facultad de Negocios en la UPC, 2017.

De acuerdo a la primera hipótesis específica; la relación entre la motivación de logro y la comprensión de un problema con funciones lineales de los estudiantes de Fundamentos para el Cálculo del turno tarde de la Facultad de Negocios en la UPC 2017, es directa y significativa; el valor de $\alpha$ es 0.05 , llamado nivel de significación teórica, lo cual corresponde al nivel de confianza con que se trabaja que es un $95 \%$.

Tabla 2

Coeficiente de correlación entre la motivación de logro y la comprensión del problema con funciones lineales en los estudiantes de fundamentos para el cálculo del turno tarde de la facultad de Negocios en la UPC 2017

\begin{tabular}{lcc}
\hline & & Comprensión del problema \\
\hline \multirow{2}{*}{ Motivación de logro } & Correlación de Spearman & -.001 \\
& Sig. (p) & .990 \\
\hline Fuente: Base de datos & $\mathrm{N}$ & 76 \\
\hline
\end{tabular}

No existe relación entre ambas variables.

Debido a que el valor obtenido por el coeficiente de correlación de Spearman es $\mathrm{p}=0.990$ y supera al valor de significación teórica $\alpha=0.05$, no se no se rechaza la 
hipótesis nula. Esto demuestra que no existe relación directa y significativa entre la motivación de logro y la comprensión del problema con funciones lineales en los estudiantes de Fundamentos para el Cálculo del turno tarde de la Facultad de Negocios en la UPC, 2017.

De acuerdo a la segunda hipótesis específica; la relación entre la motivación de logro y la concepción de un plan con funciones lineales de los estudiantes de Fundamentos para el Cálculo del turno tarde de la Facultad de Negocios en la UPC 2017, es directa y significativa; el valor de $\alpha$ es 0.05 , llamado nivel de significación teórica, lo cual corresponde al nivel de confianza con que se trabaja que es un $95 \%$.

Tabla 3

Coeficiente de correlación entre la motivación de logro y la concepción de un plan con funciones lineales en los estudiantes de fundamentos para el cálculo del turno tarde de la facultad de Negocios en la UPC 2017

\begin{tabular}{lcc}
\hline & & Concepción de un plan \\
\hline \multirow{3}{*}{ Motivación de logro } & Correlación de Spearman & -.117 \\
& Sig. $(p)$ & .315 \\
& $\mathrm{~N}$ & 76 \\
\hline
\end{tabular}

No existe relación entre ambas variables.

Debido a que el valor obtenido por el coeficiente de correlación de Spearman es $\mathrm{p}=0.315$ supera al valor de significación teórica $\alpha=0.05$, no se rechaza la hipótesis nula. Esto demuestra que no existe relación directa y significativa entre la motivación de logro y la concepción de un plan con funciones lineales en los estudiantes de Fundamentos para el Cálculo del turno tarde de la Facultad de Negocios en la UPC, 2017.

Con respecto a la tercera hipótesis específica; la relación entre la motivación de logro y la ejecución del plan con funciones lineales de los estudiantes de fundamentos para el Cálculo del turno tarde de la facultad de Negocios en la UPC 2017, es directa y significativa; el valor de $\alpha$ es 0.05 , llamado nivel de significación teórica, lo cual corresponde al nivel de confianza con que se trabaja que es un $95 \%$.

Tabla 4

Coeficiente de correlación entre la motivación de logro y la ejecución del plan con funciones lineales en los estudiantes de fundamentos para el cálculo del turno tarde de la facultad de Negocios en la UPC 2017

\begin{tabular}{llc}
\hline & & Ejecución del plan \\
\hline Motivación de logro & Correlación de Spearman & -.032
\end{tabular}




\begin{tabular}{lcc} 
& Sig. $(p)$ & .782 \\
& $\mathrm{~N}$ & 76 \\
\hline Fuente: Base de datos & &
\end{tabular}

No existe relación entre ambas variables.

Debido a que el valor obtenido por el coeficiente de correlación de Spearman es $\mathrm{p}=0.782$ supera al valor de significación teórica $\alpha=0.05$, no se rechaza la hipótesis nula. Esto demuestra que no existe relación directa y significativa entre la motivación de logro y la ejecución del plan con funciones lineales en los estudiantes de Fundamentos para el Cálculo del turno tarde de la Facultad de Negocios en la UPC, 2017.

En cuanto a la cuarta hipótesis; la relación entre la motivación de logro y la visión retrospectiva con funciones lineales de los estudiantes de fundamentos para el Cálculo del turno tarde de la facultad de Negocios en la UPC 2017, es directa y significativa; el valor de $\alpha$ es 0.05 , llamado nivel de significación teórica, lo cual corresponde al nivel de confianza con que se trabaja que es un $95 \%$.

\section{Tabla 5}

Coeficiente de correlación entre la motivación de logro y la visión retrospectiva con funciones lineales en los estudiantes de fundamentos para el cálculo del turno tarde de la facultad de Negocios en la UPC 2017

\begin{tabular}{lcc}
\hline & & Visión retrospectiva \\
\hline \multirow{2}{*}{ Motivación de logro } & Correlación de Spearman & -.107 \\
& Sig. $(p)$ & .356 \\
\hline Fuente: Base de datos & $\mathrm{N}$ & 76 \\
\hline
\end{tabular}

No existe relación entre ambas variables.

Debido a que el valor obtenido por el coeficiente de correlación de Spearman es $\mathrm{p}=0.356$ y supera al valor de significación teórica $\alpha=0.05$, no se rechaza la hipótesis nula. Esto demuestra que no existe relación directa y significativa entre la motivación de logro y la visión retrospectiva con funciones lineales en los estudiantes de Fundamentos para el Cálculo del turno tarde de la Facultad de Negocios en la UPC, 2017.

\section{Conclusiones}

Según los resultados obtenidos, no existe relación directa y significativa entre la motivación del logro y la resolución de problemas con funciones lineales. Se puede concluir que, para los estudiantes del curso de Fundamentos para el Cálculo, una buena motivación extrínseca, intrínseca o ansiedad facilitadora del rendimiento no influyen en 
la obtención de la competencia matemática de resolución de problemas. Esto se debe a que, para obtener dicha competencia se requiere de la formación de habilidades y conocimientos matemáticos, más que las ganas de querer resolver un problema.

Los resultados muestran que no existe relación directa y significativa entre la motivación del logro y la comprensión de un problema. Se puede concluir que, a pesar de tener estudiantes motivados o desmotivados no garantiza una buena comprensión del problema. Los docentes pueden aplicar estrategias para mejorar la motivación de un estudiante, pero esto no significa necesariamente una mejora de lectura o interpretación del problema. Más que eso, se necesita manejo de terminologías matemáticas, estrategias de solución y organización para una mejor comprensión.

Se demostró que la relación directa y significativa entre la motivación del logro y la concepción de un plan no existe. Esto significa que ambas son independientes. Dentro de esta investigación se ha hablado de la importancia de la concepción de un plan, proceso que se requiere de tener una buena planificación y un alto nivel analítico, creativo e innovador. La motivación del logro no garantiza una mejora en estas capacidades, más bien, esto se genera durante los primeros años escolares y se refuerzan durante los periodos universitarios.

Se determinó que no existe relación directa y significativa entre la motivación del logro y la ejecución de un plan. Se puede concluir que una buena motivación no garantiza la efectividad al momento de realizar los cálculos y aplicar el plan que se ha ideado en el paso anterior. Este proceso requiere una mayor concentración y verificación de las operaciones que se van realizando, hay que tener un buen nivel numérico y una precisión de cálculo, guardando una secuencia lógica en cada paso realizado.

Según los resultados estadísticos, no existe relación directa y significativa entre la motivación del logro y la visión retrospectiva. Por ello, para obtener una buena visión retrospectiva, cerrando así el proceso de aprendizaje, no se requiere necesariamente de una buena motivación. Muchos estudiantes son capaces de resolver un problema y más aún comprender y analizar los resultados obtenidos en problema, pero muestran que no tienen una buena motivación. Eso significa que lo hacen no porque quieran hacerlo, sino porque saben cómo hacerlo.

\section{Referencias}

Atkinson, J. (1964). An Introduction to Motivation, Nueva York - EE.UU.: Van Nostrand Reinhold. 
Ayllón, M. F. (2012). Invención-Resolución de problemas por alumnos de educación primaria en formación. Tesis doctoral, Universidad de Granada, España.

Ayllón, M.F. y Gómez, I.A y Ballesta, J. (2016). Desarrollo del pensamiento matemático y la creatividad a través de la invención y resolución de problemas. Propósitos y Representaciones 4 (1), 169-218. doi: http://dx.doi.org/10.20511/pyr2016. v4n1.89

Bengoechea, G. (1996). Bases psicopedagógicas de la educación especial. Oviedo España: Universidad de Oviedo, Servicio de Publicaciones.

Blanco, L. (1993). Una clasificación de problemas matemáticos, Sevilla - España: Épsilon n. 25.

Boscan, M. y Klever, k. (2012). Metodología basada en el método heurístico de Polya para el aprendizaje de la resolución de problemas matemáticos. Revista científica, Escenario Vol. 10. Recuperado de https://goo.g1/N8fhSN.

Chóliz, M. (2004). Psicología de la Motivación: el proceso motivacional, Valencia España: Editorial de la universidad de Valencia.

Erazo, A. (2015). La motivación y la enseñanza de la matemática en los estudiantes de los octavo, noveno y décimo grado de educación general básica de la escuela "indoamérica", ubicado en la parroquia canchagua - cantón saquisili - provincia de cotopaxi, en el año lectivo 2013 - 2014. Ambato - Ecuador.

Gasco, J. y Villarroel, J.D (2014). La motivación en la resolución de problemas aritmético-algebraicos: Un estudio con alumnado de Educación Secundaria. Electronic Journal of Research in Educational Psychology, 12(1), 83-106.

Gonzales, J. (2012). Metodologías de la investigación diseños no experimentales. Revista científica de investigación. Recuperado de https://goo.gl/kUw76m.

Iriarte, A. y Sierra, I. (2011). Estrategias metacognitivas en la resolución de problemas matemáticos. Montería - Colombia: Fondo editorial Universidad de Córdova.

McClelland, D. (1953). The achievement motive. New York - EE.UU.: AppletonCentury-Crofts.

McClelland, D. (1984). Human Motivation. Oakland, New York - EE.UU.: Scott Foresman.

McClelland, D. y Pilon, D. (1983). Sources of adult motives in patterns of parent behavior in early childhood. Journal of Personality and Social Psychology. New York EE.UU.: Scott Foresman.

Murray, H. (1938). Explorations in personality. Nueva York - EE.UU.: Oxford University Press.

Navea, A. (2015). Un estudio sobre la motivación y estrategias de aprendizaje en estudiantes universitarios de ciencias de la salud. Madrid: España.

Núñez, J. (2009). Motivación, aprendizaje y rendimiento académico. Trabajo presentado en el X Congreso Internacional Galego-Português de Psicopedagogía. Revista científica de Psicología. Recuperado de https://goo.gl/1qW7ct.

OECD (2014). Results: What students know and can do: Student Performance in Problem Solving. Paris - Francia: PISA, OECD.

OECD (2015). Resulted PISA claves. Revista científica de resultados PISA. Recuperado de https://www.oecd.org/pisa/pisa-2015-results-in-focus-ESP.pdf. 
Polya, G. (1965). Cómo plantear y resolver problemas, de Julián Zagazagoitia. México D.F. - México: Trillas.

Ramos, C. (2015). Los paradigmas de la investigación científica, Scientific research paradigms. Revista científica psicológica. Recuperado de https://www.researchgate.net/publication/282731622.

Ruíz, G y Quintana, A. (2016). Atribución de motivación de logro y rendimeinto académico e matemática. PsiqueMag 4(1), 81- 96

Sánchez, H. y Reyes, C. (2015). Metodología y diseños en la investigación científica. quinta edición. Lima - Perú: Business Support. Aneth.

Soriano, M. (2001). La motivación, pilar básico de todo tipo de esfuerzo. Revista de relaciones laborales. Recuperado de https://dialnet.unirioja.es/servlet/articulo?codigo=209932. 